\title{
EMPRESAS PÚBLICAS: TRANSFORMACIONES, DESAFÍOS Y OPORTUNIDADES: SEMINARIO INTERNACIONAL, MONTEVIDEO, OCTUBRE DE 2012
}

\author{
Daniel Chavez \\ Transnational Institute, Holanda \\ chavez@tni.org
}

Entre el 29 de octubre y el 1 de noviembre de 2012, se realizó en Montevideo el seminario internacional Empresas Públicas: Transformaciones, Desafíos y Oportunidades, coorganizado por el Transnational Institute (TNI), la Dirección Nacional de Industrias (DNI-MIEM) y la Administración Nacional de Telecomunicaciones (ANTEL) del Uruguay, en base a exposiciones a cargo de más de 30 autoridades gubernamentales, directivos y técnicos de empresas estatales, sindicalistas e investigadores académicos de diversos países del mundo.

El seminario posibilitó el intercambio de experiencias y la discusión en profundidad del significado y el rol de las empresas públicas en un contexto internacional de grandes cambios a escala regional y global. Durante los tres días del seminario, se manifestó un consenso general en torno a la significación positiva de las empresas del Estado como instrumentos de crecimiento económico y de desarrollo social, en base a la provisión de servicios públicos y un rol activo en la política industrial. Se presentaron ejemplos de empresas exitosas en países latinoamericanos gobernados por fuerzas políticas de izquierda o progresistas -Argentina, Brasil, Bolivia, Ecuador, Uruguay y Venezuela- y en Costa Rica, un país que hoy tiene un gobierno conservador, pero donde el Estado ha asumido históricamente un rol preponderante. También se analizó la trayectoria de las empresas públicas en Europa, Asia y América del Norte, y sus perspectivas en el contexto de la actual crisis mundial.

\section{LA SIGNIFICACIÓN DE LAS EMPRESAS PÚBLICAS EN AMÉRICA LATINA Y EN OTRAS REGIONES DEL MUNDO}

La sesión inaugural del seminario fue abierta por el Ministro de Industria, Energía y Minería del Uruguay, Roberto Kreimerman, quien argumentó que "las empresas públicas son una oportunidad para el desarrollo nacional porque permiten generar innovación y desarrollo en los distintos sectores", aludiendo al "gran empuje que tuvo Uruguay al formar sus empresas 
públicas”. El Ministro destacó que una de ellas, la Administración Nacional de Usinas y Transmisiones Eléctricas (UTE) -la entidad responsable de la provisión de energía eléctrica-, está celebrando su $100^{\circ}$ aniversario y afirmó que en su origen estas empresas "se crearon con una visión no muy diferente de la promovida por el actual gobierno progresista, para cumplir con un servicio estratégico que tiene que dar la nación y para ser una herramienta fuerte del desarrollo nacional". Desde la perspectiva del gobierno uruguayo, las empresas públicas "deben funcionar bien, ser eficientes, innovadoras, con calidad y productividad, pero al ser empresas del Estado también tienen que propender al desarrollo económico y a la inclusión social". "Añadió el Ministro que:

Pensamos que las empresas del Estado, aparte de su rol clave en áreas como las telecomunicaciones, el agua y la energía, tienen también que ser-bajo una política nacional, a diferencia de lo que ocurría en décadas pasadas- un instrumento de construcción de un proyecto de sociedad para América Latina y nuestro país, mejor que el que hemos tenido y a través del cual ahora debemos seguir avanzando.

En la sesión de apertura también se analizó la situación de las empresas públicas más allá de América Latina. Massimo Florio, profesor de la Universidad de Milán, presentó un detallado análisis de la experiencia europea, concluyendo que "después de dos décadas de reformas orientadas al mercado todavía es posible observar en Europa múltiples dificultades para que los pobres puedan acceder a los servicios públicos, con más de 35.000.000 de personas en situación de pobreza”. En este contexto, el investigador italiano señaló que la propiedad estatal de las empresas de telecomunicaciones, gas y electricidad "con mucha frecuencia significa costos más bajos, mejor calidad de servicios y consumidores más satisfechos", demostrando así la necesidad de "repensar el rol del Estado en la provisión de bienes y servicios públicos”.

Desde una perspectiva similar, Luc Bernier, profesor de la Escuela Nacional de Administración Pública de Quebec, analizó las experiencias de Canadá y de Estados Unidos en el contexto de la actual crisis económica y financiera, argumentando la necesidad de reevaluar el rol del Estado y las características específicas de la gestión. "Las empresas públicas pueden ser eficientes y competitivas si son administradas y controladas de la manera correcta", y también "pueden ser actores de primera línea en la economía mundial; basta ver lo que hace China con sus empresas estatales", argumentó Bernier. 


\section{LAS EMPRESAS PÚBLICAS Y LA POLÍTICA INDUSTRIAL}

Por su parte, otra alta autoridad gubernamental uruguaya, el Subsecretario de Economía y Finanzas Luis Porto, aseveró en el panel centrado en la política industrial y la promoción del desarrollo económico que "si bien es cierto que las empresas multinacionales tienen mayor productividad", las pequeñas y medianas empresas (PYMES) locales ofrecen "una utilización de factores que implican una distribución del ingreso más equitativa", las empresas cooperativas "tienen un potencial de desarrollo del capital social superior", y las empresas públicas "tienen mayor capacidad de derramar al conjunto de la sociedad".

Haciendo referencia al enfoque teórico estructuralista sobre a la relación centro-periferia promovido en su origen por la Comisión Económica para América Latina y el Caribe (CEPAL), Porto argumentó que el rol de las empresas públicas en los países del Sur debe ser "explotar al máximo las ventajas que tienen para desarrollar capacidades locales que otras firmas, como las empresas multinacionales, jamás van a desarrollar". En este sentido, el viceministro manifestó su discrepancia "con muchas propuestas de política industrial en América Latina que se han basado en atraer inversión extranjera directa como la manera de aumentar el nivel de productividad en la economía, cuando en realidad deberían ser las empresas públicas las articuladoras, dada la posibilidad que tienen de investigar y desarrollar capacidades locales". Porto subrayó también que "las empresas públicas son una oportunidad para el desarrollo de los países porque permiten generar innovación y desarrollo en muy distintos sectores".

En resumen, el Viceministro distinguió tres áreas de integración en las que las empresas públicas "tienen un rol preponderante a futuro": la regional, la nacional y la social. A nivel de la integración regional, Porto destacó la importancia que podrían asumir las empresas públicas en la optimización de sinergias en las cadenas de suministros, poniendo como ejemplo la construcción de parques eólicos construidos de forma conjunta por las empresas estatales de energía de Uruguay y Brasil.

En este mismo sentido, el Decano de la Facultad de Ingeniería de la Universidad de la República (UdelaR) del Uruguay, Héctor Cancela, afirmó que "las empresas públicas deberían reforzar su papel de liderazgo cultural" en términos de "manejo de la gestión de calidad, de los estándares y del armado de las cadenas logísticas", resaltando la importancia de las empresas del Estado en "la formación de capital humano" y en el desarrollo de nuevas opciones y especializaciones de docencia y áreas de investigación en cooperación con la universidad. 
En el mismo panel, Guillermo Guajardo, investigador del Centro de Investigaciones Interdisciplinarias en Ciencias y Humanidades de la Universidad Nacional Autónoma de México (UNAM), basado en el estudio comparativo de diversas experiencias de industrialización en América Latina, alertó sobre la necesidad de ir más allá de la economía neoclásica en el diseño de la política industrial. Tomando como ejemplo el caso mexicano, recordó que "en términos generales la política de controles a la inflación y falta de autonomía financiera ahogaron a las empresas del Estado durante la década de 1980, además de dejarse a un lado la política industrial”.

Profundizando en el análisis del rol de las empresas públicas en la política industrial, el Director de la Unidad de Cooperación Económica e Integración de la UNCTAD (la Conferencia de las Naciones Unidas sobre Comercio y Desarrollo) resaltó la necesidad de "un Estado fuerte, preocupado por la transformación y el desarrollo a partir de la industrialización”. Por su parte, el economista Manuel Montes, del South Centre, analizó la nueva realidad de las empresas estatales en la nueva coyuntura mundial, expresando que "el regreso a la época de empresas del Estado tendientes al despilfarro es poco probable" y que "las empresas estatales eficientes y competitivas podrían ser la norma en un futuro próximo". No obstante, Montes señaló también "el peligro de que se imponga una teoría ahistórica, inexacta y errónea sobre el lugar que las empresas estatales deberían asumir en la promoción del desarrollo", ya que es posible observar en países del Sur "reformas a menudo promovidas por bien intencionados movimientos sociales que abogan por la eliminación de las empresas estatales como instrumentos de desarrollo". Desde la perspectiva del representante del South Centre, "la productividad nacional debe ser modernizada y diversificada sobre una base de empresas estatales fuertes", ya que estas "pueden desempeñar un papel fundamental en el logro de los objetivos de desarrollo industrial".

\section{LAS EMPRESAS DEL ESTADO Y LOS SERVICIOS PÚBLICOS}

Los representantes de empresas públicas de Uruguay, Venezuela y Costa Rica, destacaron los aportes de estas entidades a distintos niveles: en la dinamización del desarrollo económico endógeno, en la defensa de la soberanía nacional, en la promoción de la innovación y el avance científico y tecnológico, en la generación de infraestructura de calidad, y en la provisión de servicios de calidad desde una perspectiva de equidad e inclusión social.

La empresa anfitriona del seminario, ANTEL, expuso su condición de liderazgo en el sector de las telecomunicaciones. En un país que ya ha alcanzado índices de cobertura universal de los servicios telefónicos, la 
empresa estatal uruguaya lidera el mercado de telefonía móvil con el 47\% de participación, en un contexto de mercado liberalizado y de competencia con los dos conglomerados transnacionales privados hegemónicos en la región: el Grupo Telefónica/Movistar (de matriz española) y el Grupo Carso/ América Móvil/Claro (de matriz mexicana). ANTEL está desarrollando ahora un proyecto de extensión de la fibra óptica sin tasa de conexión a todos los hogares del país, lo cual permitirá acceder a múltiples de servicios (Internet de alta velocidad, televisión digital, etc.) con la mejor calidad. La empresa estatal uruguaya ha asumido como definición estratégica la conexión del 100\% de los hogares del país a Internet (al presente, el 48\% de los hogares ya están conectados a la red), así como "la implantación de la mejor tecnología celular para la transmisión de datos a través del primer y más avanzado despliegue en América Latina de banda ancha móvil de última generación" (LTE y 4G), de acuerdo a la presentación realizada por Javier Emicuri, Gerente de Soluciones Empresariales de ANTEL.

La realidad de las empresas públicas en el sector de las telecomunicaciones también fue abordada por un ingeniero de la Compañía Anónima Nacional Teléfonos de Venezuela (CANTV), Julio Yánez, quien explicó las responsabilidades asumidas por el Estado en la universalización de los servicios públicos en el marco del proceso bolivariano. Después de caracterizar el acceso a las telecomunicaciones como un derecho humano fundamental, Yáñez expuso los cambios procesados por la CANTV desde su renacionalización y los logros obtenidos en términos de mayor seguridad tecnológica, soberanía e independencia. Durante los últimos cinco años, la gestión de la empresa

[...] se ha orientado a rescatar y recuperar a la CANTV como la empresa de telecomunicaciones del Estado, en manos privadas desde el año 1999 y recuperada en año 2007, democratizando los procesos de planificación y de toma de decisiones y procurando atender las necesidades de los sectores sociales tradicionalmente excluidos.

En la actualidad, explicó el investigador venezolano,

[...] se ha avanzado en la transición de empresa privada a empresa estatal y está surgiendo una empresa socialista de nuevo tipo, revirtiendo la tercerización de servicios, reduciendo la dependencia tecnológica, e integrando la empresa a los planes estratégicos de desarrollo nacional.

Otras dos empresas estatales uruguayas activas en la provisión de servicios públicos en el Uruguay que expusieron en el seminario fueron UTE y Obras Sanitarias del Estado (OSE). UTE es la empresa responsable 
del nivel de cobertura prácticamente universal (99\%) alcanzado por el país. De acuerdo a la exposición realizada por Eduardo Bergerie, Gerente de Gestión Distribución de Energía Eléctrica, la empresa estatal uruguaya lidera los indicadores de servicios en América Latina en base a "una política de mejora continua de procesos asociada a la introducción de nuevas tecnologías y a la constante profesionalización del personal”. En relación a los desafíos de futuro, Bergerie presentó los planes para mejorar la gestión, aumentar la eficiencia energética, y avanzar en la generación distribuida y en base a fuentes renovables, entre otros proyectos en curso.

El Gerente General de OSE, Danilo Ríos, resaltó la "visión de desarrollo" de "una empresa pública de excelencia, comprometida con la prestación de servicios de agua potable y saneamiento, que procura el acceso universal y la satisfacción del usuario de forma eficiente y sustentable, con responsabilidad social, ambiental y participación ciudadana”. También destacó el hecho que Uruguay es el primer país del mundo que ha declarado el acceso al agua potable y al saneamiento como derecho humano fundamental, a partir de la reforma constitucional aprobada por la ciudadanía en el año 2004. De acuerdo a los datos proporcionados por la empresa, Uruguay cuenta hoy con un nivel de cobertura de $94 \%$ del total de la población, que asciende al $98 \%$ en las áreas urbanas.

En base a los principios de universalización de los servicios e inclusión social, OSE está trabajando actualmente proyectos de abastecimiento en pequeñas localidades rurales, en un plan de regularización en asentamientos urbanos informales, en la reducción del agua no contabilizada, y en el desarrollo de Unidades Potabilizadoras de Agua (UPA) portables.

El panel centrado en servicios públicos también tuvo en cuenta la necesidad de promover nuevas y más significativas instancias de cooperación entre empresas del Estado. En este sentido, el redactor de esta síntesis, Daniel Chavez, del TNI, cuestionó la fascinación que todavía se observa en muchos países del Sur en torno a las asociaciones públicoprivadas (PPP, por su sigla en inglés), sin tener en cuenta la evidencia de experiencias fallidas acumulada en los países originalmente promotores de este tipo de arreglos comerciales. Como alternativa, se propuso discutir las posibilidades ofrecidas por las asociaciones público-público $(\mathrm{PuP})$, una modalidad de cooperación que ya demostrado su potencial (así como sus limitaciones y el riesgo de que también sean 'cooptadas' por el mercado) en el sector del agua y que podría ser extendida a otros sectores. Afirmó el expositor:

Es preciso apostar a las PuPs aprovechando por ejemplo las posibilidades que podría ofrecer un espacio como la Organización 
Internacional de Telecomunicaciones de las Américas (OITA), una prometedora instancia de cooperación recientemente conformada por las empresas estatales de telecomunicaciones de Argentina, Bolivia, Brasil, Cuba, Ecuador, Paraguay, Uruguay y Venezuela.

El profesor Alberto Cortés Ramos, de la Universidad de Costa Rica, destacó la necesidad de defender a las empresas públicas de las constantes amenazas de privatización y de liberalización. Después de explicar la larga tradición costarricense de servicios públicos de calidad y con equidad, así como la alta valoración de las empresas del Estado que los ciudadanos siguen expresando en las encuestas, Cortés Ramos advirtió que:

[...] en las últimas tres décadas, grupos políticos y económicos que no creen en lo público han promovido la transformación de la matriz del Estado, causando no sólo cambios en la gestión y la racionalidad de funcionamiento de las empresas públicas con la introducción de una lógica empresarial y aumentos de tarifas, sino también un deterioro de la calidad de los servicios.

Ante esta realidad, Cortés Ramos argumentó a favor de "incorporar más participación ciudadana, no sólo en la rendición de cuentas, sino también en el proceso de formulación y seguimiento de las políticas públicas". También propuso "recuperar y actualizar la visión de largo plazo que históricamente ha caracterizado a las empresas del Estado más exitosas, incorporando también una visión que tenga en cuenta la dimensión regional, de cooperación Sur-Sur". Por último, el investigador costarricense propuso "una alianza estratégica entre las empresas públicas y el sector académico para profundizar en la investigación de la realidad específica de estas empresas y contribuir a la generación de alternativas progresistas".

\section{EL POLISÉMICO SIGNIFICADO DE LO PÚBLICO}

Desde Argentina, el investigador del Centro de Investigaciones Participativas en Políticas Económicas y Sociales (CIPPES), Alfredo Schclarek Curutchet, argumentó la necesidad de:

[...] democratizarelgobiernodelasempresaspúblicastransparentando las actividades y decisiones, fortaleciendo la cooperación y el control al interior del Estado, y fomentando la gestión participativa a través de la inclusión de la sociedad civil en las juntas directivas.

Desde la perspectiva sindical, el Presidente de la Federación de Funcionarios de OSE (FFOSE), Carlos Sosa, argumentó la necesidad de rediscutir el significado del concepto de empresa pública en la realidad 
uruguaya y latinoamericana contemporánea. En este sentido, recordó que las movilizaciones de los trabajadores y de otras organizaciones de la sociedad civil posibilitaron en el año 2004 la reforma de la constitución del país, cuyo artículo 47 establece que "el servicio público de saneamiento y el servicio público de abastecimiento de agua para consumo humano serán prestados exclusiva y directamente por personas jurídicas estatales". Desde una perspectiva similar, Susan Spronk, profesora de la Universidad de Ottawa, había destacado en la sesión inaugural del seminario la necesidad de permanecer alerta ante la introducción de "modalidades encubiertas de privatización”, aludiendo al avance de la tercerización y de otras formas de externalización de servicios en empresas públicas uruguayas y de otros países de la región.

Alvaro Portillo, profesor de Sociología Urbana en la Facultad de Arquitectura de la Universidad de la República, se refirió al "Estado en transformación” que se aprecia hoy en Uruguay, en Venezuela y en otros países de América Latina. "El Estado no es una entidad monolítica, sino que es una relación social, dinámica, compleja e internamente contradictoria, existiendo una correlación entre la forma dominante de acumulación capitalista y la forma estatal", argumentó Portillo. Desde esta perspectiva, sería necesario incorporar en el análisis de las empresas públicas no sólo a las empresas responsables activas en el sector productivo a responsables del abastecimiento de servicios públicos, sino también a las empresas estatales del sector financiero. "En el Uruguay, el Banco de la República es hoy el principal apoyo financiero de la producción nacional y uno de los principales apoyos al consumo". Para optimizar el impacto de las empresas públicas, Portillo insistió que "se requiere una profunda coordinación entre las empresas públicas a nivel de planificación y de operaciones, y también de coordinación a nivel territorial, desarrollando estrategias conjuntas". Por último, Portillo destacó las posibilidades ofrecidas por la integración de las empresas públicas a escala regional, "aprovechando las posibilidades de acuerdos de complementación" ofrecidas por la multiplicidad de gobiernos latinoamericanos que comparten visiones políticas progresistas.

En varias presentaciones realizadas por investigadores académicos de diversos países también se realizaron profundas críticas al paradigma de la Nueva Gestión Pública (NGP) en el ámbito de las empresas. En base al análisis de experiencias canadienses y europeas, Luc Bernier manifestó que "la NGP nunca tuvo los resultados que habían sido prometidos", por lo cual es necesario repensar la gestión de las empresas del Estado: "existe la posibilidad de transformar la gobernanza de las empresas; la propiedad pública puede ser eficiente si es realizada de la manera apropiada”. Bernier también fue categórico al afirmar que "la crisis mundial del año 2008 ha 
demostrado que estas empresas podrían ser muy útiles instrumentos de política pública”.

Pedro Narbondo, Director del Instituto de Ciencia Política de la Universidad de la República, centró su exposición en "dos factores claves para que las empresas públicas sigan siendo un instrumento de desarrollo y de construcción de igualdad social". El investigador uruguayo destacó, en primer lugar, "la necesidad de que las empresas públicas sigan estando efectivamente subordinadas al Estado y a la conducción gubernamental representativa del conjunto de la ciudadanía" y, en segundo lugar, "la importancia de la correcta articulación de esa conducción gubernamental con cuerpos profesionales estables y profesionalizados, valga la redundancia”. Narbondo argumentó que las perspectivas neoliberales y neogerencialistas hicieron que por mucho tiempo se dejara de percibir "la importancia de las empresas públicas como mecanismos para avanzar hacia un modelo de desarrollo con mayor valor agregado y mayor equidad social que el ofrecido por el mercado", por lo cual hoy es imprescindible "que las empresas públicas actúen con una lógica desmercantilizada". La desmercantilización de la gestión pública, en particular en la provisión de servicios públicos, también fue señalada como una necesidad insoslayable por David McDonald, profesor de la Universidad de Queens y Codirector del Proyecto Servicios Municipales (Municipal Services Project, MSP).

El investigador canadiense subrayó la necesidad de discutir el problema de la corporatización de las empresas públicas, haciendo referencia a "entidades que legalmente siguen siendo de titularidad pública pero que en la práctica funcionan como empresas privadas, al haber asumido como propios los principios de gestión y administración basados en el mercado". En base a la evidencia empírica proporcionada por investigaciones desarrolladas por el MSP en las áreas de agua, salud y energía eléctrica alrededor del mundo, McDonald afirmó que:

[...] la corporatización es como un lobo con piel de cordero, que ofrece una fachada de propiedad pública mientras propaga la ideología de mercado y la acumulación privada, y todo ello sin tener que asumir los riesgos económicos y políticos asociados a las formas tradicionales de privatización.

\section{EL DEBATE EN TORNO AL NEOEXTRACTIVISMO Y EL NEODESARROLLISMO}

La significación y el rol de las empresas públicas en la nueva coyuntura política y económica de América Latina, y en particular su relación con otros debates en torno a los conceptos de neoextractivismo y 
neodesarrolismo, también fueron temas discutidos en el seminario de Montevideo. De acuerdo a la presentación realizada por Atilio Boron, Director del Programa Latinoamericano de Educación a Distancia en Ciencias Sociales (PLED), se aprecia en la actualidad la ascendencia de un enfoque "excesivamente crítico", que presenta a algunas políticas públicas de los gobiernos progresistas de la región:

[...] como expresiones de un mismo proceso de adaptación a las nuevas circunstancias creadas por la fase más reciente del desarrollo del capitalismo a nivel internacional, que de alguna manera obligaría a los países de la periferia a extremar la explotación de los recursos naturales agravando al mismo tiempo la desindustrialización.

Según Boron, "es válido y necesario criticar al extractivismo, pero no se puede afirmar que sea un fenómeno contemporáneo o específico de algunos gobiernos". La exposición concluyó con una invitación al debate:

¿Qué pueden hacer los gobiernos interesados en elevar el nivel de equidad y de bienestar social con los recursos naturales de los que están tan bien dotados? No debemos olvidar que América Latina es la región del mundo más rica en recursos naturales, pero también la que tiene la mayor desigualdad social.

Desde una perspectiva diferente, el economista Carlos Arze, del Centro de Estudios para el Desarrollo Laboral y Agrario (CEDLA), presentó "una crítica a la política desarrollista y extractivista del actual gobierno boliviano", exponiendo las contradicciones teóricas y discursivas "del socialismo comunitario como meta del desarrollo", recordando que su principal ideólogo -el actual vicepresidente del país- había planteado originalmente que el objetivo del Gobierno sería "implantar un capitalismo andinoamazónico”. En base a múltiples datos estadísticos, Arze argumentó que:

[...] el Estado no está redistribuyendo en la misma medida en que está aumentando la renta derivada de los recursos naturales, la que representa apenas una tercera parte del valor de las remesas que los trabajadores migrantes envían a sus familias.

También se refirió a las renacionalizaciones que han tenido lugar en Bolivia:

[...] en el sector hidrocarburos, efectivamente se ha incrementado la renta que percibe el Estado: desde un 25\% en el año 2004 hasta el $65 \%$ actual. Sin embargo, el $50 \%$ de esa recuperación fue aprobado en una ley del año 2005 y no en el gobierno de Evo Morales. El 
restante $15 \%$ surge de utilidades por contratos con empresas transnacionales, las que siguen haciendo un muy buen negocio en Bolivia.

Arze concluyó su exposición afirmando que:

[...] las empresas extranjeras han bajado su tasa de ganancia, pero no la masa de su ganancia, ya que en la actualidad el Estado apenas controla el $18 \%$ de la producción de hidrocarburos, mientras que las transnacionales (incluyendo a Petrobras) siguen en control del $82 \%$.

Desde Colombia, Lyda Fernanda Forero, investigadora del Transnational Institute, argumentó que es necesario:

[...] dejar de pensar en alternativas de desarrollo y pasar a cuestionar el propio significado del "desarrollo", en el marco de una crisis civilizatoria que no es sólo económica, sino que es también una crisis ambiental, energética y del modo de producción en su conjunto.

El debate cardinal planteado por la economista colombiana fue resumido en una pregunta crucial: “`cómo superar la idea de crecimiento permanente sin que eso implique un deterioro de las condiciones de vida?”.

Daniel Kersffeld, Coordinador de la Maestría en Gestión Pública del Instituto de Altos Estudios Nacionales (IAEN) del Ecuador, inició su exposición planteando que el debate sobre las empresas públicas, el desarrollo y el extractivismo implica en esencia repensar el significado del Estado. Poniendo como ejemplo el caso ecuatoriano, Kersffeld señaló que hay un dilema entre "un Estado que se autodefine como el Estado de la revolución ciudadana y que por lo tanto está interesando en reconocer y ampliar los derechos de poblaciones previamente marginadas", y "la necesidad que tiene el Estado de insertarse estratégicamente en la economía mundial, debiendo apelar a recursos que posee en gran cantidad". Ante esta situación, Kersffeld advirtió que los Estados de signo progresista deben asumir decisiones complejas y hasta contradictorias: "potenciar, frenar o revertir las tendencias tradicionales de desarrollo; promover inversiones en opciones de desarrollo alternativo; revitalizar el potencial para la cooperación multilateral en la protección ambiental”, entre otras.

El Director del Centro de Investigación y Prevención de la Criminalidad Económica (CIPCE), Pedro Biscay, realizó una exposición centrada en las perspectivas de la sociedad civil en torno a las acciones de las empresas, tanto públicas como privadas, y la defensa de los derechos sociales y económicos. El investigador argentino subrayó que: 
[...] la discusión sobre el neoextractivismo y el neodesarrollismo no debe estar limitada al accionar de las empresas extractivas, sino que también es preciso recordar que estos conceptos están muy relacionados a la renta financiera, y en particular a la especulación financiera.

Biscay argumentó que:

[...] no es posible discutir las opciones de futuro de las empresas públicas sin tener en cuenta la correlación histórica que en la Argentina y en otros países latinoamericanos ha existido entre la matriz neoliberal y una forma particular de organización de la producción y las finanzas.

Javier Taks, profesor de la Universidad de la República, cerró el panel sobre estos temas sugiriendo dos temas para futuros debates: "En primer lugar, la ontología de la relación sociedad-naturaleza tan propia del modernismo, planteando la posibilidad de reconocimiento de otras ontologías alternativas. En segundo lugar, rediscutir el significado de la matriz de lo público en las sociedades latinoamericanas contemporáneas”.

\section{LASEMPRESASPÚBLICASENELURUGUAYCONTEMPORÁNEO}

La mesa redonda de clausura del seminario contó con la participación de los presidentes de las cinco empresas públicas más importantes del Uruguay: Jorge Setelich (AFE), Carolina Cosse (ANTEL), Raúl Sendic (ANCAP), Milton Machado (OSE) y Gonzalo Casaravilla (UTE). El panel fue coordinado por el Director Nacional de Industrias, Sebastián Torres, quién sostuvo que las empresas del Estado inciden en el desarrollo nacional en varios planos: "el control de recursos naturales, el desarrollo de las regiones más atrasadas, la disponibilidad de bienes esenciales, el ahorro de divisas, la generación de empleo y el desarrollo tecnológico", estableciendo "la base material que define la soberanía, la equidad y el desarrollo de un país”.

$\mathrm{Al}$ analizar la importancia de este tipo de empresas, Torres explicó que no se puede prescindir del concepto de lo público, el cual a su juicio "no debe ser entendido como antagónico al mercado, sino como algo que lo trasciende". "Las empresas públicas requieren de un liderazgo y una coordinación, y por lo tanto deben visualizarse desde el colectivo y no desde la perspectiva del individuo", subrayó. Torres ańadió que:

[...] las organizaciones son las piedras fundamentales de la sociedad, no los individuos atomizados y aislados tomando decisiones en base a mecanismos de mercado. Eso hay que comprenderlo porque 
cambia lo que pensamos sobre la economía: es la lógica de la acción colectiva la que determina los procesos de desarrollo en el mediano y largo plazo.

Por su parte la Presidenta de ANTEL, Carolina Cosse, recordó que la sociedad uruguaya tuvo que enfrentar en el pasado reciente diversos intentos de privatización del patrimonio público, y que la ciudadanía decidió que las áreas estratégicas de la economía nacional debían permanecer en manos del Estado. También destacó dos dimensiones del aporte de las empresas estatales al desarrollo: en primer lugar, brindar servicios de calidad con sensibilidad social y, en segundo lugar, ser un factor fundamental del desarrollo productivo. Seńaló Cosse:

Necesitamos recordar cómo y por qué fueron creadas estas empresas. Las decisiones sobre infraestructura que están adoptando hoy las empresas públicas sobre la diversificación de la matriz energética (UTE), el desarrollo de nuevas fuentes de energía y la producción de alimentos (ANCAP) y la extensión de la fibra óptica al hogar (ANTEL), tendrán un impacto que se extenderá durante las próximas tres décadas.

Una perspectiva similar fue expuesta por el Presidente de ANCAP, Raúl Sendic, quien argumentó que "en una empresa pública uruguaya lo primero que hay que discutir es la estrategia: que papel tiene la empresa en la economía y en relación al desarrollo productivo y social del país". Después de enumerar los múltiples cambios procesados al interior de ANCAP en la última década, Sendic destacó la importancia de establecer "una alianza estrecha con la investigación, con la academia y con la innovación permanente. Las empresas públicas deben promover y deben financiar la investigación científica en el país”. El presidente de ANCAP informó que eso ya está haciendo desde la empresa que el preside:

[...] ya tenemos acuerdos con la Universidad de la República, con la Agencia Nacional de Investigación e Innovación (ANII), y ahora estamos en conversaciones con el Instituto Clemente Estable. Al mismo tiempo, también estamos promoviendo la búsqueda y el desarrollo de talentos internos al interior de la empresa.

La significación histórica de las empresas públicas en la sociedad y en la economía del Uruguay ya había sido reconocida en la sesión de apertura del seminario por el profesor Gerardo Caetano, de la Universidad de la República. "El caso uruguayo está constituido por una matriz de empresas públicas muy arraigado que ha resistido los embates privatizadores y neoliberales que en otros países del continente han devastado al Estado", 
explicó Caetano, recordando que "en el Uruguay hace más de 100 años que se debate sobre la debilidades y las fortalezas de esa matriz".

El historiador uruguayo argumentó "que ya no es válida aquella idea primigenia de que lo público es sólo lo estatal" y que "hoy debemos considerar y analizar distintas dimensiones de lo público". Por otra parte, señaló que:

[...] tampoco podemos olvidar que el país ha pasado de una tasa de inversión de un $10 \%$ a una tasa de inversión productiva de más de un $22 \%$ en los últimos diez años, fundamentalmente a través de inversión extranjera directa, lo cual constituye toda una revolución. Y esta nueva realidad le plantea a las empresas públicas de un país como el Uruguay muchos y profundos desafíos renovados.

\section{PERSPECTIVAS DE TRABAJO EN CONJUNTO}

El seminario fue concebido por las tres organizaciones convocantes -TNI, ANTEL y DNI-MIEM - no como una actividad aislada, sino como parte de un proceso internacional más amplio de debate, de intercambio y de investigación sobre el presente y el futuro de las empresas públicas en el actual contexto mundial.

En este sentido, durante los tres días del encuentro y en particular en las dos sesiones finales del tercer día, se propusieron diversas ideas para dar continuidad al proceso iniciado en Montevideo. La relevancia de un proceso de este tipo fue destacada por Fiona Dove, Directora del Transnational Institute, quien señaló que:

[...] las diversas presentaciones realizadas en este seminario demuestran la importancia de seguir trabajando en torno al tema de las empresas públicas en este momento particular de la economía mundial. Debemos potenciar el trabajo en conjunto de quienes estamos motivados por el desarrollo humano y sustentable y no sólo por la búsqueda del crecimiento económico.

La Presidente de ANTEL anunció en el panel de cierre que las tres organizaciones convocantes publicarán un libro con las presentaciones ofrecidas por los diversos expositores. TNI, ANTEL y la DNI-MIEM ya están trabajando en esa dirección. Los técnicos de ANTEL le proporcionaron al Transnational Institute los archivos de audio y de texto de todas las presentaciones, y TNI ya se ha comunicado con los expositores para coordinar sus contribuciones a la mencionada publicación. Está previsto que el libro se publique en español y en inglés durante 2013. Se 
espera que la publicación constituya un insumo útil y relevante para otros encuentros internacionales de debate e intercambio sobre la temática de las empresas públicas previstos para el ańo próximo.

En el seminario de Montevideo participaron dos integrantes del Comité Científico del Centro Internacional de Investigación e Información sobre la Economía Social, Pública y Cooperativa (CIRIEC), una red global de investigadores sobre empresas públicas y temas afines. Los profesores Massimo Florio y Luc Bernier forman parte del comité de coordinación de un proyecto internacional de investigación sobre The Future of Public Enterprises: Mission, Performance and Governance (El Futuro de las Empresas Públicas: Misión, Desempeño y Gobernanza), basado en una propuesta metodológica orientada a la sistematización de experiencias y el análisis comparativo de empresas de diversos países y sectores.

El profesor Massimo Florio detalló el contexto, los objetivos y la metodología del proyecto de investigación del CIRIEC. El diseño del proyecto asume que: "las herramientas analíticas utilizadas tradicionalmente para examinar el desempeño de las empresas públicas no son apropiadas para dar cuenta de las transformaciones recientes del entorno económico y social", que "la crisis económica actual hace que sea aun más importante entender cómo las empresas públicas podrían contribuir al crecimiento económico", y que "organizaciones internacionales, como la OCDE, el Banco Mundial y las Naciones Unidas están una vez más interesadas en este tema”.

Los objetivos del proyecto están orientados a:

[...] abrir un debate sobre las empresas públicas y su contribución al crecimiento y el desarrollo; actualizar la teoría normativa existente sobre este tipo de empresas; investigar el impacto de la liberalización del mercado en el rendimiento y la misión de las empresas públicas; comprender los factores clave del éxito o fracaso de las empresas públicas en relación a sus objetivos y en términos de mayor bienestar social; y crear una red internacional y multidisciplinaria de expertos encaminada a investigar el nuevo papel y la contribución a la sociedad de las empresas públicas.

En el marco del seminario se manifestó el interés de varios investigadores uruguayos en crear el Capítulo Uruguay del CIRIEC, a los efectos de desarrollar nuevos estudios centrados en la realidad específica de las empresas públicas del país. Ello implicaría promover un nuevo espacio de confluencia de investigadores, autoridades gubernamentales y directivos de las principales empresas públicas uruguayas. 
Una de los iniciativas principales a ser impulsadas por el capítulo uruguayo del CIRIEC sería la realización de un proyecto de investigación a ser desarrollado en el transcurso del año próximo. A tales efectos, se adaptarían las propuestas metodológicas de CIRIEC, del MSP, del TNI y de otras redes activas en este campo a la realidad concreta del Uruguay.

La otra iniciativa internacional de investigación en curso presentada en el seminario fue el Municipal Services Project. El profesor David McDonald explicó la razón de ser del MSP: "un programa de investigación que explora alternativas a la privatización y a la comercialización de los servicios públicos en las áreas de electricidad, salud, agua y saneamiento en África, Asia y América Latina”. Las actividades del MSP están basadas en el trabajo en red "de académicos, sindicatos, organizaciones no gubernamentales y movimientos sociales de todas las regiones del mundo". Mientras que las dos primeras fases del proyecto (2000-2007) se centraron en las críticas a la privatización, el proyecto está focalizado en la actualidad "en el análisis de los resultados y las condiciones de sostenibilidad y de replicabilidad de modelos alternativos de prestación de servicios (no sólo a nivel municipal)”.

Varios de los investigadores que expusieron en el seminario plantearon la necesidad de retomar la investigación sobre la realidad de las empresas públicas de América Latina, dada la generalizada ausencia de trabajos académicos actualizados en este campo. En el caso específico del Uruguay, se informó sobre estudios en curso sobre algunas empresas, centrados fundamentalmente en una perspectiva de historia económica. Una realidad similar se observa en otros países de la región, donde se aprecia la necesidad de complementar este tipo de estudios con aportes de otras disciplinas, incluyendo una sistematización en profundidad de la situación actual de las empresas y de sus perspectivas de evolución en el futuro.

Varios de los panelistas en el seminario de Montevideo también destacaron la altísima relevancia e importancia de la investigación, el debate y el intercambio de experiencias sobre el presente y el futuro de las empresas públicas en el actual contexto de crisis mundial. En particular, los representantes de la UNCTAD (Richard Kozul-Wright) y del South Centre (Manuel Torres) manifestaron su interés en apoyar nuevas instancias de cooperación y trabajo en conjunto en este campo. En este sentido, en el seminario se consideró informalmente la posible organización un segundo seminario en el año 2013, profundizando en los temas para el debate planteados en Montevideo. En términos más concretos, desde el South Centre se sugirió la posibilidad de organizar un seminario en Asia, preferentemente coorganizado en conjunto con empresas públicas de esa región. 
Otra de las posibles áreas de trabajo en conjunto más a largo plazo es la cooperación Sur-Sur. En este sentido, el Director de la Agencia Uruguaya de Cooperación (AUCI), Martín Rivero, señaló que "las empresas públicas tienen un gran potencial para mejorar las condiciones del desarrollo", agregando que "los servicios públicos constituyen un componente fundamental para la creación de la infraestructura apropiada y necesaria para el desarrollo humano". Las capacidades del país anfitrión para ofrecer cooperación técnica en este campo específico fueron destacadas por Rivero, al afirmar que las empresas estatales del Uruguay:

[...] tienen una visión universalista y de promoción integral de las capacidades sociales; en algunas empresas de manera muy explícita y en otras de manera un tanto menos evidente, pero expresando en todos los casos una perspectiva de desarrollo que constituye un valor de la experiencia uruguaya que podría ser ofrecido como propuesta de cooperación hacia otros países de la región y del mundo.

El seminario pudo ser seguido no sólo por el público uruguayo que estaba presente en el centro de conferencias de ANTEL, ya que Adinet.TV, el canal en Internet de la empresa estatal de telecomunicaciones, transmitió en directo todas las presentaciones durante las tres jornadas del seminario.

Al cierre del seminario, el Director Nacional de Industrias, Sebastián Torres, agradeció a todos los participantes y expresó que:

[...] esperamos que todo este esfuerzo sea retomado en otras iniciativas. Pensamos en generar nuevos encuentros de este tipo, tal vez no en Montevideo y si en algún otro país de América Latina; incluso se ha planteado la posibilidad de realizar un seminario similar a este en Asia.

En síntesis, concluyó Torres:

[...] queremos reforzar esta red que se está creando en estos días, y asumimos el desafío muy grande de seguir trabajando juntos sobre un tema que, si bien está muy presente en la agenda pública, no había contado hasta ahora con espacios de debate y de intercambio de ideas con la misma calidad que se pudo apreciar en Montevideo. 